\title{
Analysis of Heating Effects and Different Wave Forms on Peristaltic Flow of Carreau Fluid in Rectangular Duct
}

\author{
Safia Akram (iD) ${ }^{1}$ and Najma Saleem $(\mathbb{D})^{2}$ \\ ${ }^{1}$ MCS, National University of Sciences and Technology, Islamabad, Pakistan \\ ${ }^{2}$ College of Sciences \& Human Studies, Prince Mohammad Bin Fahd University, Al Khobar 31952, Saudi Arabia \\ Correspondence should be addressed to Safia Akram; drsafiaakram@gmail.com
}

Received 3 February 2020; Accepted 25 March 2020; Published 20 May 2020

Academic Editor: Ruben Specogna

Copyright (c) 2020 Safia Akram and Najma Saleem. This is an open access article distributed under the Creative Commons Attribution License, which permits unrestricted use, distribution, and reproduction in any medium, provided the original work is properly cited.

\begin{abstract}
The existing analysis deals with heat transfer occurrence on peristaltic transport of a Carreau fluid in a rectangular duct. Flow is scrutinized in a wave frame of reference moving with velocity $c$ away from a fixed frame. A peristaltic wave propagating on the horizontal side walls of a rectangular duct is discussed under lubrication approximation. In order to carry out the analytical solution of velocity, temperature, and pressure gradient, the homotopy perturbation method is employed. Graphical results are displayed to see the impact of various emerging parameters of the Carreau fluid and power law index. Trapping effects of peristaltic transport is also discussed and observed that number of trapping bolus decreases with an increase in aspect ratio $\beta$.
\end{abstract}

\section{Introduction}

The applications of peristaltic flows in medical and engineering sciences have attracted the attention of a number of researchers. Applications of peristalsis occur in swallowing food through the esophagus, urine transport from the kidney to the bladder through the ureter, transport of the spermatozoa in the efferent ducts of the male reproductive tract, movement of the ovum in the fallopian tube, movement of the chyme in the gastrointestinal tract, the transport of lymph in the lymphatic vessels, and vasomotion in the small blood vessels such as the arterioles, veins, and capillaries. The peristaltic phenomenon was first discussed by Latham [1]. Based on his experimental theory, numerous researchers have inspected the phenomenon of peristaltic transport under many conjectures [2-10].

Another fascinating area in connection with peristaltic motion is the heat transfer which has industrial applications like sanitary fluid transport, blood pumps in the heart-lungs machine and transport of corrosive fluids where the contact of fluid with the machinery parts are prohibited. Only a limited attention has been focused to the study of peristaltic flows with heat transfer [11-15].

An immense amount of literature is presented on twodimensional peristaltic flow problems. The study of peristaltic phenomenon in a rectangular channel was first examined by [16]. Based on the theory of [16], several researchers have studied the phenomenon of peristaltic transport in a rectangular duct under various approximations [17-23]. In the papers cited above, the phenomena of heat transfer are not taken into account. Keeping in mind the present information, the heat transfer phenomena on the peristaltic flow of non-Newtonian fluid have not been discussed in a threedimensional channel. So, the aim of the present problem is to discuss the effects of heat transfer on peristaltic flow of a non-Newtonian fluid in a rectangular duct with different wave forms. The governing equations for the three-dimensional rectangular channel are first modeled for Carreau fluid and then simplified under the long wavelength and low Reynolds number approximation. Homotopy perturbation technique is carried out to calculate the analytical solution of the highly nonlinear partial differential equations. The expressions for 
velocity, pressure rise, pressure gradient, and temperature have been computed and discussed through graphs. Threedimensional graphical representations for the velocity field are also discussed through graphs. In the end, different wave forms are also used.

\section{Mathematical Formulation of the Problem}

Let us consider the peristaltic flow of an incompressible Carreau fluid in a duct of rectangular cross section having the channel width $2 \mathrm{~d}$ and height $2 \mathrm{a}$. We are considering the Cartesian coordinates system in such a way that the $X$-axis is taken along the axial direction, $Y$-axis is taken along the lateral direction, and $Z$-axis is along the vertical direction of a rectangular duct.

The peristaltic waves on the walls are represented as

$$
Z=H(X, t)= \pm a \pm b \cos \left[\frac{2 \pi}{\lambda}(X-c t)\right]
$$

where $a$ and $b$ are the amplitudes of the waves, $\lambda$ is the wave length, $c$ is the velocity of propagation, $t$ is the time, and $X$ is the direction of wave propagation. The walls parallel to the $X Z$ plane remain undisturbed and are not subjected to any peristaltic wave motion. We assume that the lateral velocity is zero as there is no change in the lateral direction of the duct cross section. Let $(U, 0, W)$ be the velocity for a rectangular duct. The governing equations for the flow problem are

$$
\begin{aligned}
& \frac{\partial U}{\partial X}+\frac{\partial W}{\partial Z}=0 \\
& \rho\left(\frac{\partial U}{\partial t}+U \frac{\partial U}{\partial X}+W \frac{\partial U}{\partial Z}\right) \\
& =-\frac{\partial P}{\partial X}+\frac{\partial}{\partial X} S_{X X}+\frac{\partial}{\partial Y} S_{X Y}+\frac{\partial}{\partial Z} S_{X Z}, \\
& 0=-\frac{\partial P}{\partial Y}+\frac{\partial}{\partial X} S_{Y X}+\frac{\partial}{\partial Y} S_{Y Y}+\frac{\partial}{\partial Z} S_{Y Z}, \\
& \rho\left(\frac{\partial W}{\partial t}+U \frac{\partial W}{\partial X}+W \frac{\partial W}{\partial Z}\right) \\
& =-\frac{\partial P}{\partial Z}+\frac{\partial}{\partial X} S_{Z X}+\frac{\partial}{\partial Y} S_{Z Y}+\frac{\partial}{\partial Z} S_{Z Z}, \\
& \rho C^{\prime}\left(\frac{\partial T}{\partial t}+U \frac{\partial T}{\partial X}+W \frac{\partial T}{\partial Z}\right) \\
& =K^{\prime}\left(\frac{\partial^{2} T}{\partial X^{2}}+\frac{\partial^{2} T}{\partial Y^{2}}+\frac{\partial^{2} T}{\partial Z^{2}}\right)+S_{X X} \frac{\partial U}{\partial X}++S_{Y X} \frac{\partial U}{\partial Y} \\
& \quad+S_{X Z}\left(\frac{\partial W}{\partial X}+\frac{\partial U}{\partial Z}\right)+S_{Y Z} \frac{\partial W}{\partial Y}+S_{Z Z} \frac{\partial W}{\partial Z},
\end{aligned}
$$

in which $\rho$ is the density, $P$ is the pressure, $t$ is the time, and $S$ ' $s$ is the stress tensor for the Carreau fluid; $C^{\prime}$ is the specific heat; and $T$ is the temperature. The stress tensor for the Carreau fluid is defined by [17].

$$
\mathbf{S}=\mu\left(1+(\Gamma \dot{\boldsymbol{\gamma}})^{2}\right)^{(n-1) / 2} \dot{\boldsymbol{\gamma}}
$$

Let us define a wave frame $(x, y)$ moving with the velocity caway from the fixed frame $(X, Y)$ by the transformation

$$
\begin{aligned}
x & =X-c t, \\
y & =Y, \\
z & =Z, \\
u & =U-c, \\
w & =W, \\
p(x, z) & =P(X, Z, t) .
\end{aligned}
$$

Defining the following nondimensional quantities,

$$
\begin{aligned}
& \bar{x}=\frac{x}{\lambda}, \\
& \bar{y}=\frac{y}{d}, \\
& \bar{z}=\frac{z}{a}, \\
& \bar{u}=\frac{u}{c}, \\
& \bar{w}=\frac{w}{c \delta}, \\
& \bar{t}=\frac{c t}{\lambda}, \\
& h=\frac{H}{a}, \\
& \bar{p}=\frac{a^{2} p}{\mu c \lambda}, \\
& \bar{S}_{\bar{y} \bar{y}}=\frac{\lambda}{\mu c} S_{y y} . \\
& \bar{S}_{\bar{y} \bar{z}}=\frac{d}{\mu c} S_{y z}, \\
& \bar{S}_{\bar{x} \bar{y}}=\frac{\lambda}{\mu c} S_{z z}, \\
& \beta=\frac{\rho a c \delta}{\mu}, \\
& \beta=\frac{a}{d}, \\
& \bar{S}_{x y}, \\
& \bar{S}_{x z}, \\
&
\end{aligned}
$$


Using the above nondimensional quantities in Equations (2), (3), (4), (5) and (7), the resulting equations after dropping the bars can be written as

$$
\begin{aligned}
& \frac{\partial u}{\partial x}+\frac{\partial w}{\partial z}=, \\
& \operatorname{Re}\left(u \frac{\partial u}{\partial x}+w \frac{\partial u}{\partial z}\right) \\
&=-\frac{\partial p}{\partial x}+\delta \frac{\partial}{\partial x} \tau_{x x}+\beta^{2} \frac{\partial}{\partial y} S_{x y}+\frac{\partial}{\partial z} S_{x z} \\
& 0=-\frac{\partial p}{\partial y}+\delta^{2} \frac{\partial}{\partial x} S_{y x}+\delta^{2} \frac{\partial}{\partial y} S_{y y}+\delta \frac{\partial}{\partial z} S_{y z}, \\
& \operatorname{Re} \delta^{2}\left(u \frac{\partial w}{\partial x}+w \frac{\partial w}{\partial z}\right) \\
&=-\frac{\partial p}{\partial z}+\delta^{2} \frac{\partial}{\partial x} S_{z x}+\delta \beta^{2} \frac{\partial}{\partial y} S_{z y}+\delta^{2} \frac{\partial}{\partial z} S_{z z}, \\
& \operatorname{Re} \delta\left(u \frac{\partial \theta}{\partial x}+w \frac{\partial \theta}{\partial z}\right) \\
&=\frac{1}{\operatorname{Pr}}\left(\delta^{2} \frac{\partial^{2} \theta}{\partial x^{2}}+\beta^{2} \frac{\partial^{2} \theta}{\partial y^{2}}+\frac{\partial^{2} \theta}{\partial z^{2}}\right) \\
&+ E c \delta S_{x x} \frac{\partial u}{\partial x}+\beta^{2} S_{x y} \frac{\partial u}{\partial y}+\beta^{2} \delta S_{y z} \frac{\partial w}{\partial y} \\
&\left.+\delta^{2} S_{z z} \frac{\partial w}{\partial z}+S_{z x}\left(\delta^{2} \frac{\partial w}{\partial x}+\frac{\partial u}{\partial z}\right)\right),
\end{aligned}
$$

where

$$
\begin{aligned}
S_{x x}= & -2 \delta\left(1+\frac{(n-1)}{2} \mathrm{We}^{2} \dot{\gamma}^{2}\right) \frac{\partial u}{\partial x} \\
S_{x y}= & -\left(1+\frac{(n-1)}{2} \mathrm{We}^{2} \dot{\gamma}^{2}\right) \frac{\partial u}{\partial y}, \\
S_{x z}= & -\left(1+\frac{(n-1)}{2} \mathrm{We}^{2} \dot{\gamma}^{2}\right)\left(\frac{\partial u}{\partial z}+\delta^{2} \frac{\partial w}{\partial x}\right), \\
S_{y y}= & 0, \\
S_{y z}= & -\delta\left(1+\frac{(n-1)}{2} \mathrm{We}^{2} \dot{\gamma}^{2}\right) \frac{\partial w}{\partial y}, \\
S_{z z}= & -2\left(1+\frac{(n-1)}{2} \mathrm{We}^{2} \dot{\gamma}^{2}\right) \frac{\partial w}{\partial z}, \\
\dot{\gamma}^{2}= & 2 \delta^{2}\left(\frac{\partial u}{\partial x}\right)^{2}+\beta^{2}\left(\frac{\partial u}{\partial y}\right)^{2}+\delta^{2} \beta^{2}\left(\frac{\partial w}{\partial y}\right)^{2} \\
& +\delta^{2}\left(\frac{\partial w}{\partial z}\right)^{2}+\left(\delta^{2} \frac{\partial w}{\partial x}+\frac{\partial u}{\partial z}\right)^{2} .
\end{aligned}
$$

Under the assumption of long wavelength $\delta \leq 1$ and low Reynolds number Re $\longrightarrow 0$, Equations (10), (11), (12), (13), (14) and (21) take the form

$$
\begin{aligned}
\frac{d p}{d x}= & \beta^{2} \frac{\partial^{2} u}{\partial y^{2}}+\frac{\partial^{2} u}{\partial z^{2}}+\frac{n-1}{2} \mathrm{We}^{2} \beta^{4} \frac{\partial}{\partial y}\left(\frac{\partial u}{\partial y}\right)^{3} \\
& +\frac{n-1}{2} \mathrm{We}^{2} \frac{\partial}{\partial z}\left(\frac{\partial u}{\partial z}\right)^{3} \\
& +\frac{n-1}{2} \mathrm{We}^{2} \beta^{2} \frac{\partial}{\partial y}\left(\frac{\partial u}{\partial y}\left(\frac{\partial u}{\partial z}\right)^{2}\right) \\
& +\frac{n-1}{2} \mathrm{We}^{2} \beta^{2} \frac{\partial}{\partial z}\left(\frac{\partial u}{\partial z}\left(\frac{\partial u}{\partial y}\right)^{2}\right) . \\
\frac{\partial^{2} \theta}{\partial z^{2}}+ & \beta^{2} \frac{\partial^{2} \theta}{\partial y^{2}}+E c \operatorname{Pr}\left(\beta^{2}\left(\frac{\partial u}{\partial y}\right)^{2}+\frac{n-1}{2} \beta^{4} \mathrm{We}^{2}\left(\frac{\partial u}{\partial y}\right)^{4}\right. \\
+ & \frac{n-1}{2} \beta^{2} \mathrm{We}^{2}\left(\frac{\partial u}{\partial y}\right)^{2}\left(\frac{\partial u}{\partial z}\right)^{2}+\left(\frac{\partial u}{\partial z}\right)^{2} \\
+ & \left.\frac{n-1}{2} \mathrm{We}^{2}\left(\frac{\partial u}{\partial z}\right)^{4}+\frac{n-1}{2} \beta^{2} \mathrm{We}^{2}\left(\frac{\partial u}{\partial y}\right)^{2}\left(\frac{\partial u}{\partial z}\right)^{2}\right)=0 .
\end{aligned}
$$

The corresponding boundary conditions are

$$
\begin{aligned}
& u=-1 \text { at } y= \pm 1, \\
& u=-1 \text { at } z= \pm h(x)= \pm 1 \pm \varphi \cos 2 \pi x, \\
& \theta=0 \text { at } y=1, \\
& \theta=0 \text { at } z=h(x), \\
& \theta=1 \text { at } y=-1, \\
& \theta=1 \text { at } z=-h(x) .
\end{aligned}
$$

where $0 \leq \varphi \leq 1, \varphi=0$ for straight duct and $\varphi=1$ corresponds to total occlusion.

\section{Solution of the Problem}

3.1. Homotopy Perturbation Method. The solution of the nonlinear partial differential Equations (22) and (23) has been calculated using homotopy perturbation technique. The homotopy perturbation method for Equations (22) and (23) can be defined as [24-30].

$$
\begin{aligned}
H(v, q)= & (1-q)\left(\mathcal{E}(v)-\mathfrak{E}\left(u_{0}\right)\right)+q\left(\mathfrak{E}(v)+\beta^{2} \frac{\partial^{2} u}{\partial y^{2}}\right. \\
& +\frac{n-1}{2} \mathrm{We}^{2} \beta^{4} \frac{\partial}{\partial y}\left(\frac{\partial v}{\partial y}\right)^{3}+\frac{n-1}{2} \mathrm{We}^{2} \frac{\partial}{\partial z}\left(\frac{\partial v}{\partial z}\right)^{3} \\
& +\frac{n-1}{2} \mathrm{We}^{2} \beta^{2} \frac{\partial}{\partial y}\left(\frac{\partial v}{\partial y}\left(\frac{\partial v}{\partial z}\right)^{2}\right) \\
& \left.+\frac{n-1}{2} \mathrm{We}^{2} \beta^{2} \frac{\partial}{\partial z}\left(\frac{\partial v}{\partial z}\left(\frac{\partial v}{\partial y}\right)^{2}\right)-\frac{d p}{d x}\right)=0,
\end{aligned}
$$




$$
\begin{aligned}
H(\Theta, q)= & (1-q)\left(£(\Theta)-£\left(\tilde{\theta}_{0}\right)\right)+q\left(£(\Theta)+\beta^{2} \frac{\partial^{2} \Theta}{\partial y^{2}}\right. \\
& +E c \operatorname{Pr}\left(\beta^{2}\left(\frac{\partial v}{\partial y}\right)^{2}+\frac{n-1}{2} \beta^{4} \mathrm{We}^{2}\left(\frac{\partial v}{\partial y}\right)^{4}\right. \\
& +\frac{n-1}{2} \beta^{2} \mathrm{We}^{2}\left(\frac{\partial v}{\partial y}\right)^{2}\left(\frac{\partial v}{\partial z}\right)^{2} \\
& +\left(\frac{\partial v}{\partial z}\right)^{2}+\frac{n-1}{2} \mathrm{We}^{2}\left(\frac{\partial v}{\partial z}\right)^{4} \\
& \left.\left.+\frac{n-1}{2} \beta^{2} \mathrm{We}^{2}\left(\frac{\partial v}{\partial y}\right)^{2}\left(\frac{\partial v}{\partial z}\right)^{2}\right)\right)=0
\end{aligned}
$$

or

$$
\begin{aligned}
& H(v, q)=\mathfrak{E}(v)-\mathfrak{E}\left(u_{0}\right)+q \mathfrak{E}\left(u_{0}\right)+q\left(\beta^{2} \frac{\partial^{2} u}{\partial y^{2}}\right. \\
& +\frac{n-1}{2} \mathrm{We}^{2} \beta^{4} \frac{\partial}{\partial y}\left(\frac{\partial v}{\partial y}\right)^{3}+\frac{n-1}{2} \mathrm{We}^{2} \frac{\partial}{\partial z}\left(\frac{\partial v}{\partial z}\right)^{3} \\
& +\frac{n-1}{2} \mathrm{We}^{2} \beta^{2} \frac{\partial}{\partial y}\left(\frac{\partial v}{\partial y}\left(\frac{\partial v}{\partial z}\right)^{2}\right) \\
& \left.+\frac{n-1}{2} \mathrm{We}^{2} \beta^{2} \frac{\partial}{\partial z}\left(\frac{\partial v}{\partial z}\left(\frac{\partial v}{\partial y}\right)^{2}\right)-\frac{d p}{d x}\right)=0, \\
& H(\Theta, q)=\mathfrak{E}(\Theta)-\mathfrak{E}\left(\tilde{\theta}_{0}\right)+q £\left(\tilde{\theta}_{0}\right)+q\left(\beta^{2} \frac{\partial^{2} \Theta}{\partial y^{2}}\right. \\
& +E c \operatorname{Pr}\left(\beta^{2}\left(\frac{\partial v}{\partial y}\right)^{2}+\frac{n-1}{2} \beta^{4} \mathrm{We}^{2}\left(\frac{\partial v}{\partial y}\right)^{4}\right. \\
& +\frac{n-1}{2} \beta^{2} \mathrm{We}^{2}\left(\frac{\partial v}{\partial y}\right)^{2}\left(\frac{\partial v}{\partial z}\right)^{2} \\
& +\left(\frac{\partial v}{\partial z}\right)^{2}+\frac{n-1}{2} \mathrm{We}^{2}\left(\frac{\partial v}{\partial z}\right)^{4} \\
& \left.\left.+\frac{n-1}{2} \beta^{2} \mathrm{We}^{2}\left(\frac{\partial v}{\partial y}\right)^{2}\left(\frac{\partial v}{\partial z}\right)^{2}\right)\right)=0 \text {. }
\end{aligned}
$$

Here, $q$ is an embedding parameter which has the range $0 \leq q \leq 1$, under the condition that for $q=0$, we get the initial solution, and for $q=1$, we seek the final solution. Here, $£$ is the linear operator which is taken here as $£=\partial^{2} / \partial z^{2}$. We define the initial guess as

$$
\begin{aligned}
& u_{0}=-1+\left(h^{2}-z^{2}\right)+\frac{(1-y)}{2}-\frac{(h-z)}{2 h}, \\
& \tilde{\theta}_{0}=\left(z^{2}-h^{2}\right)+\frac{(h-z)}{2 h}+\frac{1}{\beta^{2}}\left(1-y^{2}\right) .
\end{aligned}
$$

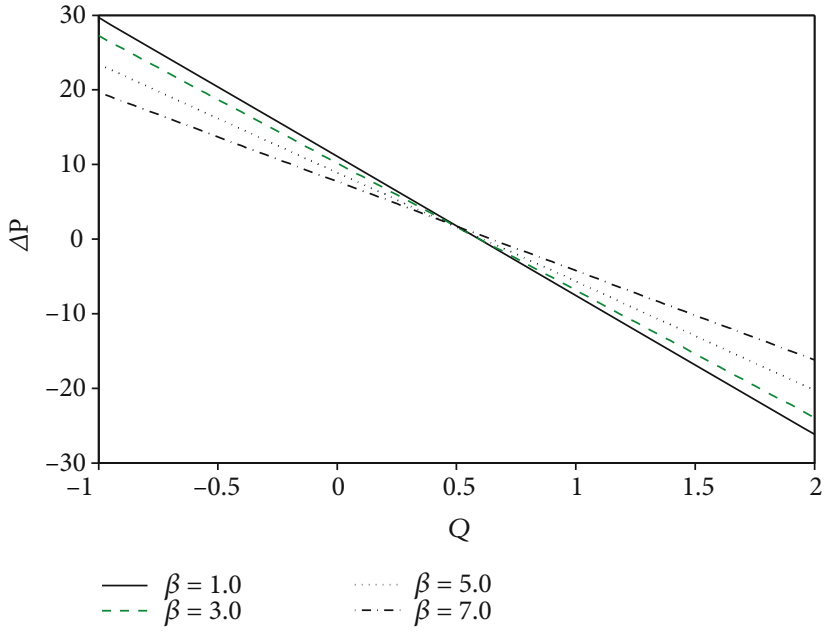

FIGURE 1: Variation of pressure rise with volume flow rate $Q$ for different values of $\beta$ for fixed values of $\varphi=0.7, \mathrm{We}=0.5$, and $n=0.6$.

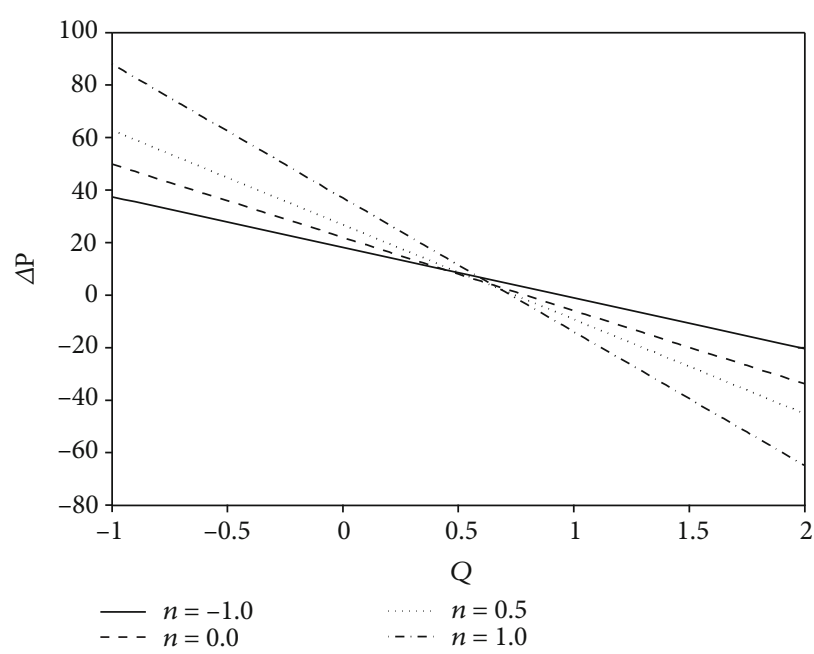

FIGURE 2: Variation of pressure rise with volume flow rate $Q$ for different values of $n$ for fixed values of $\varphi=0.8, \mathrm{We}=0.6$, and $\beta=4$.

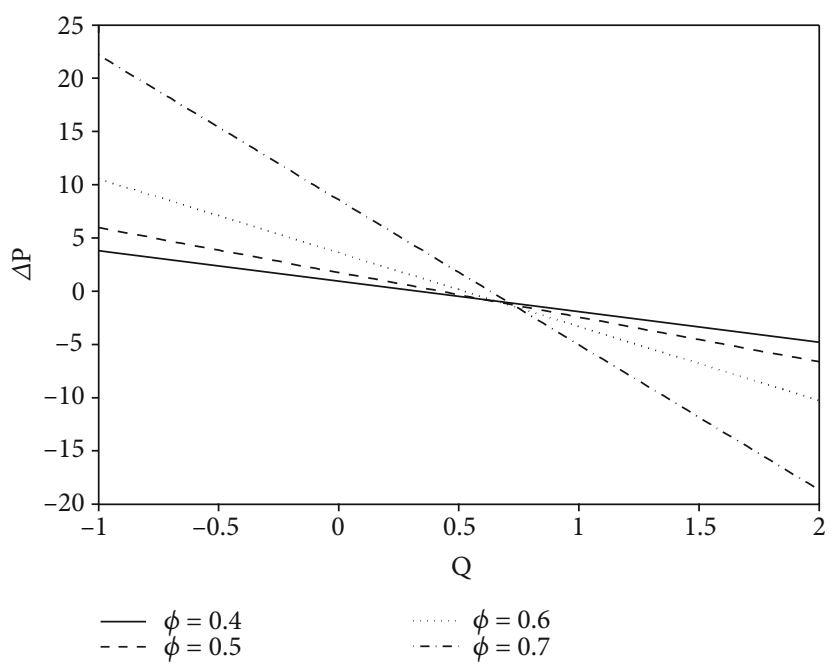

FIgURE 3: Variation of pressure rise with volume flow rate $Q$ for different values of $\varphi$ for fixed values of $n=0.5$, We $=0.6$, and $\beta=4$. 


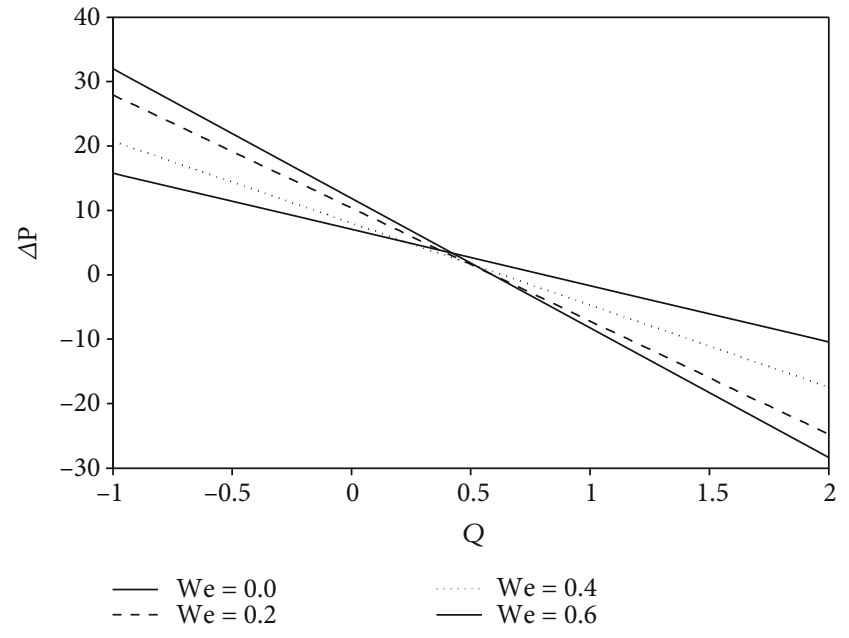

Figure 4: Variation of pressure rise with volume flow rate $Q$ for different values of We for fixed values of $\varphi=0.7, n=0.3$, and $\beta=6$.

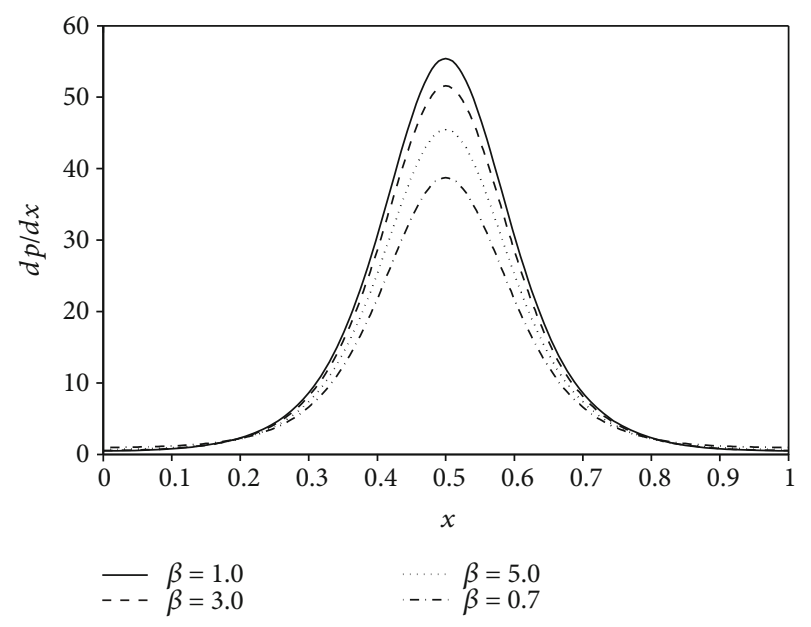

FIGURE 5: Variation of pressure gradient with $x$ for different values of $\beta$ for fixed values of $\phi=0.5, n=0.5$, We $=0.4$, and $Q=-2$.

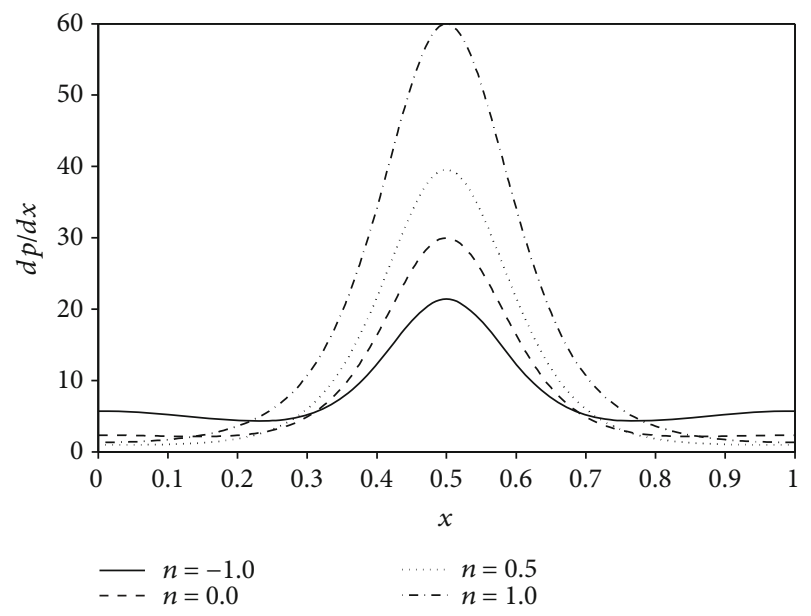

FIgURE 6: Variation of pressure gradient with $x$ for different values of $n$ for fixed values of $\varphi=0.5, \beta=4$, We $=0.6$, and $Q=-2$.

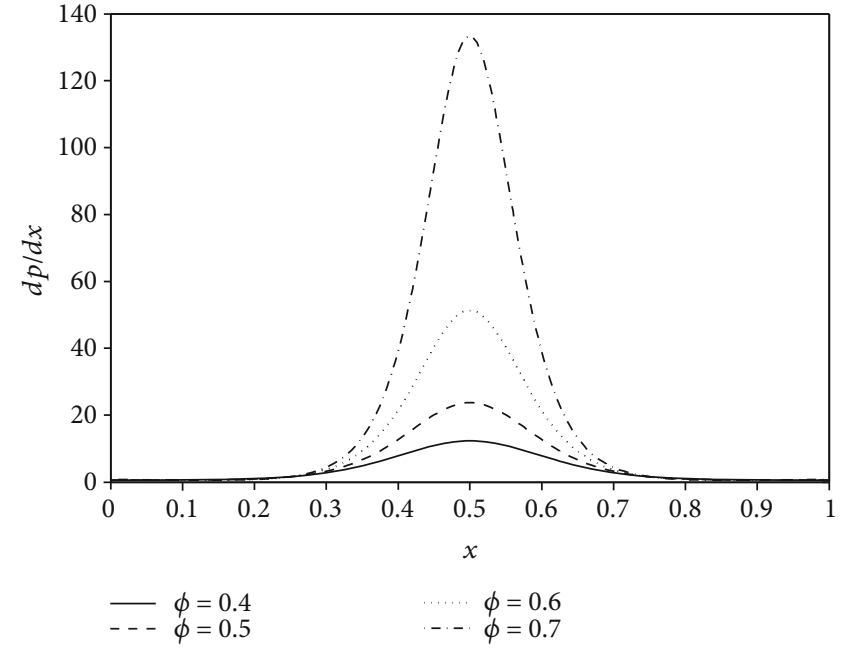

FIGURE 7: Variation of pressure gradient with $x$ for different values of $\varphi$ for fixed values of $n=0.5, \beta=4, \mathrm{We}=0.6$, and $Q=-1$.

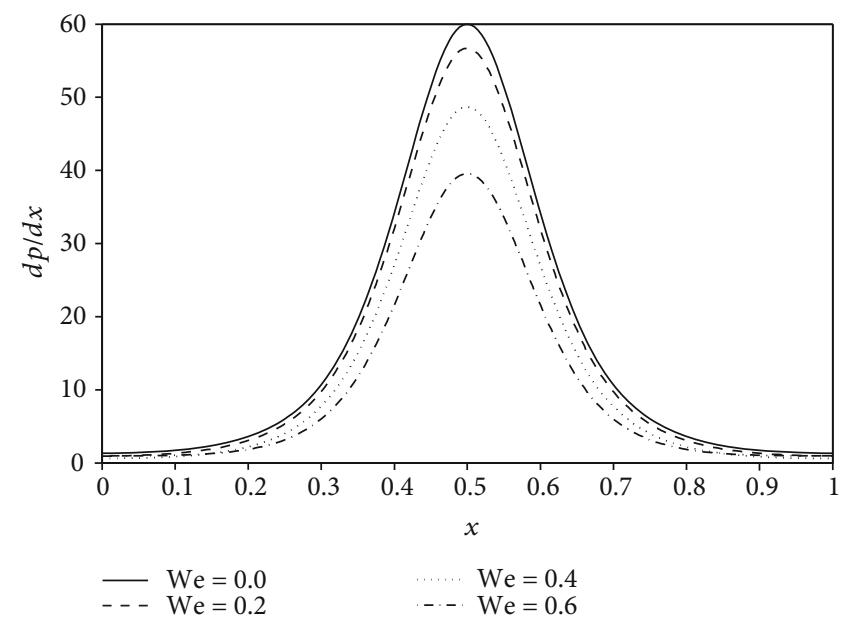

FIgURE 8: Variation of pressure gradient with $x$ for different values of We for fixed values of $\varphi=0.5, \beta=4, n=0.5$, and $Q=-2$.

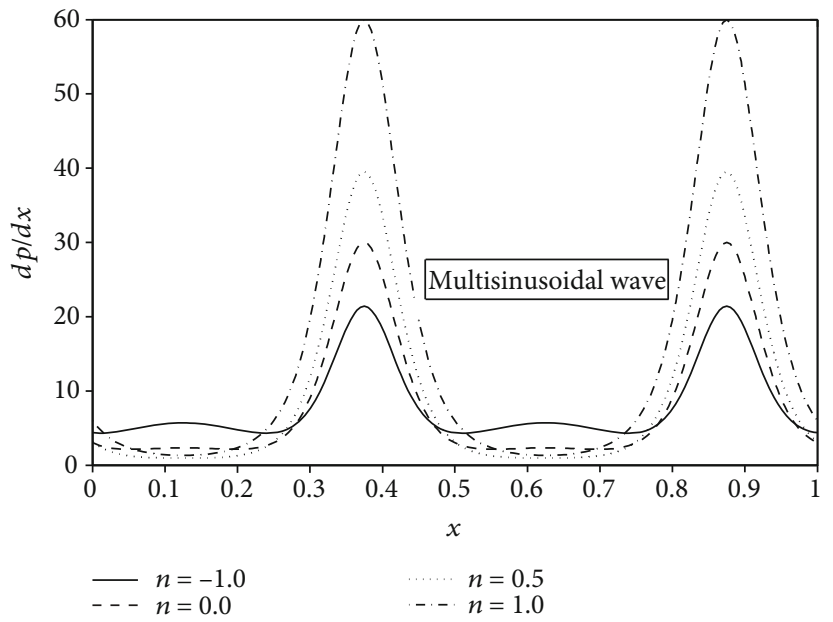

Figure 9: Variation of pressure gradient with $x$ for different values of $n$ for fixed values of $\varphi=0.5, \beta=4, \mathrm{We}=0.6$, and $Q=-2$. 
Let us define

$$
\begin{gathered}
v(x, y, z, q)=v_{0}+q v_{1}+q^{2} v_{2}+\cdots, \\
\Theta(x, y, z, q)=\Theta_{0}+q \Theta_{1}+q^{2} \Theta_{2}+\cdots,
\end{gathered}
$$

substituting Equation (30) into Equations (26) and (27), and then comparing the like powers of $q$, one obtains the following problems with the corresponding boundary conditions.

\subsection{Zeroth Order System.}

$$
\begin{aligned}
\mathfrak{E}\left(v_{0}\right)-\mathfrak{E}\left(u_{0}\right) & =0, \\
\mathfrak{E}\left(\Theta_{0}\right)-\mathfrak{E}\left(\tilde{\theta}_{0}\right) & =0, \\
v_{0} & =-1 \text { at } y= \pm 1, \\
v_{0} & =-1 \text { at } z= \pm h(x), \\
\Theta_{0} & =0 \text { at } y=1, \\
\Theta_{0} & =0 \text { at } z=h(x), \\
\Theta_{0} & =1 \text { at } y=-1, \\
\Theta_{0} & =1 \text { at } z=-h(x) .
\end{aligned}
$$

\subsection{First-Order System.}

$$
\begin{aligned}
& £\left(v_{1}\right)+£\left(u_{0}\right)+\left(\beta^{2} \frac{\partial^{2} v_{0}}{\partial y^{2}}+\frac{n-1}{2} \mathrm{We}^{2} \beta^{4} \frac{\partial}{\partial y}\left(\frac{\partial v_{0}}{\partial y}\right)^{3}\right. \\
& +\frac{n-1}{2} \mathrm{We}^{2} \frac{\partial}{\partial z}\left(\frac{\partial v_{0}}{\partial z}\right)^{3}+\frac{n-1}{2} \mathrm{We}^{2} \beta^{2} \frac{\partial}{\partial y}\left(\frac{\partial v_{0}}{\partial y}\left(\frac{\partial v_{0}}{\partial z}\right)^{2}\right) \\
& \left.+\frac{n-1}{2} \mathrm{We}^{2} \beta^{2} \frac{\partial}{\partial z}\left(\frac{\partial v_{0}}{\partial z}\left(\frac{\partial v_{0}}{\partial y}\right)^{2}\right)-\frac{d p}{d x}\right)=0, \\
& \mathfrak{E}\left(\Theta_{1}\right)+\mathfrak{E}\left(\tilde{\theta}_{0}\right)+\left(\beta^{2} \frac{\partial^{2} \Theta_{0}}{\partial y^{2}}+E c \operatorname{Pr}\left(\beta^{2}\left(\frac{\partial v_{0}}{\partial y}\right)^{2}\right.\right. \\
& +\frac{n-1}{2} \beta^{4} \mathrm{We}^{2}\left(\frac{\partial v_{0}}{\partial y}\right)^{4}+\frac{n-1}{2} \beta^{2} \mathrm{We}^{2}\left(\frac{\partial v_{0}}{\partial y}\right)^{2} \\
& \left(\frac{\partial v_{0}}{\partial z}\right)^{2}+\left(\frac{\partial v_{0}}{\partial z}\right)^{2}+\frac{n-1}{2} \mathrm{We}^{2}\left(\frac{\partial v_{0}}{\partial z}\right)^{4} \\
& \left.\left.+\frac{n-1}{2} \beta^{2} \mathrm{We}^{2}\left(\frac{\partial v_{0}}{\partial y}\right)^{2}\left(\frac{\partial v_{0}}{\partial z}\right)^{2}\right)\right)=0 \\
& v_{1}=0 \text { at } y= \pm 1 \text {, } \\
& v_{1}=0 \text { at } z= \pm h(x) \text {, } \\
& \Theta_{1}=0 \text { at } y=1 \text {, } \\
& \Theta_{1}=0 \text { at } z=h(x), \\
& \Theta_{1}=0 \text { at } y=-1 \text {, } \\
& \Theta_{1}=0 \text { at } z=-h(x) \text {. }
\end{aligned}
$$

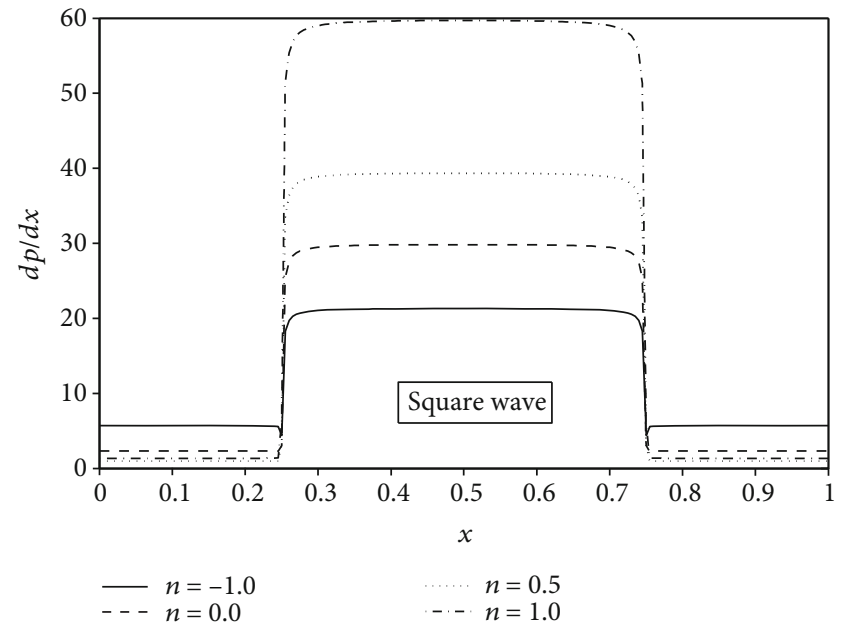

FIGURE 10: Variation of pressure gradient with $x$ for different values of $n$ for fixed values of $\varphi=0.5, \beta=4, \mathrm{We}=0.6$, and $Q=-2$.

\subsection{Second-Order System.}

$$
\begin{aligned}
& £\left(v_{2}\right)+\beta^{2} \frac{\partial^{2} v_{1}}{\partial y^{2}}+\frac{n-1}{2} \mathrm{We}^{2} \beta^{4} \frac{\partial}{\partial y}\left(3\left(\frac{\partial v_{0}}{\partial y}\right)^{2}\left(\frac{\partial v_{1}}{\partial y}\right)\right) \\
& +\frac{n-1}{2} \mathrm{We}^{2} \frac{\partial}{\partial z}\left(3\left(\frac{\partial v_{0}}{\partial z}\right)^{2}\left(\frac{\partial v_{1}}{\partial z}\right)\right) \\
& +\frac{n-1}{2} \mathrm{We}^{2} \beta^{2} \frac{\partial}{\partial y}\left(2\left(\frac{\partial v_{0}}{\partial y}\right)\left(\frac{\partial v_{0}}{\partial z}\right)\left(\frac{\partial v_{1}}{\partial z}\right)\right. \\
& \left.+\left(\frac{\partial v_{1}}{\partial y}\right)\left(\frac{\partial v_{0}}{\partial z}\right)^{2}\right)+\frac{n-1}{2} \mathrm{We}^{2} \beta^{2} \frac{\partial}{\partial z} \\
& \cdot\left(2\left(\frac{\partial v_{0}}{\partial y}\right)\left(\frac{\partial v_{0}}{\partial z}\right)\left(\frac{\partial v_{1}}{\partial y}\right)+\left(\frac{\partial v_{1}}{\partial z}\right)\left(\frac{\partial v_{0}}{\partial y}\right)^{2}\right)=0 \\
& \mathfrak{E}\left(\Theta_{2}\right)+\left(\beta^{2} \frac{\partial^{2} \Theta_{1}}{\partial y^{2}}+E c \operatorname{Pr}\left(2 \beta^{2}\left(\frac{\partial v_{0}}{\partial y}\right)\left(\frac{\partial v_{1}}{\partial y}\right)\right.\right. \\
& +\frac{n-1}{2} \beta^{4} \mathrm{We}^{2}\left(4\left(\frac{\partial v_{0}}{\partial y}\right)^{3}\left(\frac{\partial v_{1}}{\partial y}\right)\right) \\
& +2\left(\frac { n - 1 } { 2 } \beta ^ { 2 } \mathrm { We } ^ { 2 } \left(2\left(\frac{\partial v_{0}}{\partial y}\right)^{2}\left(\frac{\partial v_{0}}{\partial z}\right)\left(\frac{\partial v_{1}}{\partial z}\right)\right.\right. \\
& \left.\left.+2\left(\frac{\partial v_{0}}{\partial y}\right)\left(\frac{\partial v_{1}}{\partial y}\right)\left(\frac{\partial v_{0}}{\partial z}\right)^{2}\right)\right)+2\left(\frac{\partial v_{0}}{\partial z}\right)\left(\frac{\partial v_{1}}{\partial z}\right) \\
& \left.+\frac{n-1}{2} \mathrm{We}^{2}\left(4\left(\frac{\partial v_{0}}{\partial z}\right)^{3}\left(\frac{\partial v_{1}}{\partial z}\right)\right)\right)=0 \\
& v_{2}=0 \text { at } y= \pm 1 \text {, } \\
& v_{2}=0 \text { at } z= \pm h(x) \text {, } \\
& \Theta_{2}=0 \text { at } y=1 \text {, } \\
& \Theta_{2}=0 \text { at } z=h(x), \\
& \Theta_{2}=0 \text { at } y=-1 \text {, } \\
& \Theta_{2}=0 \text { at } z=-h(x) \text {. }
\end{aligned}
$$




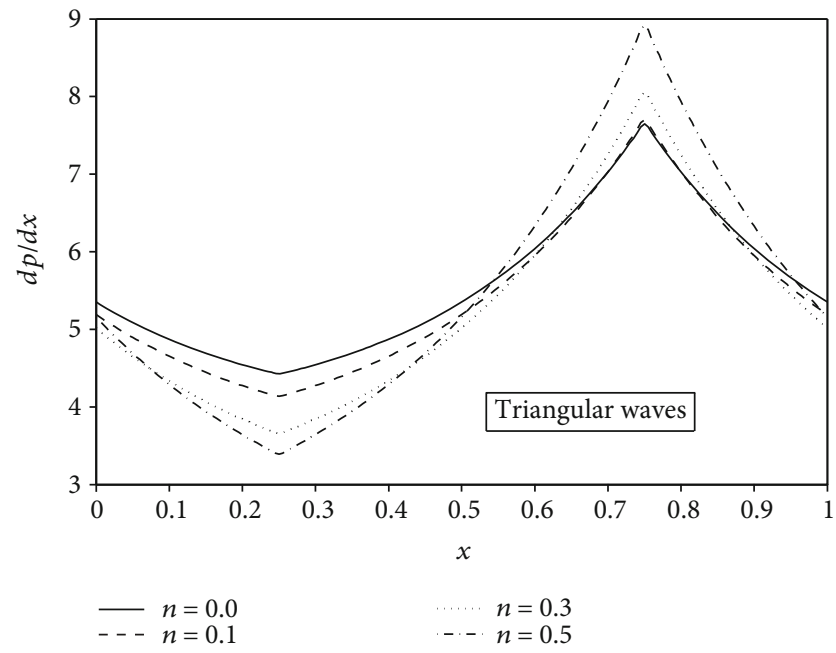

FIGURE 11: Variation of pressure gradient with $x$ for different values of $n$ for fixed values of $\varphi=0.5, \beta=6$, We $=0.6$, and $Q=-3.5$.

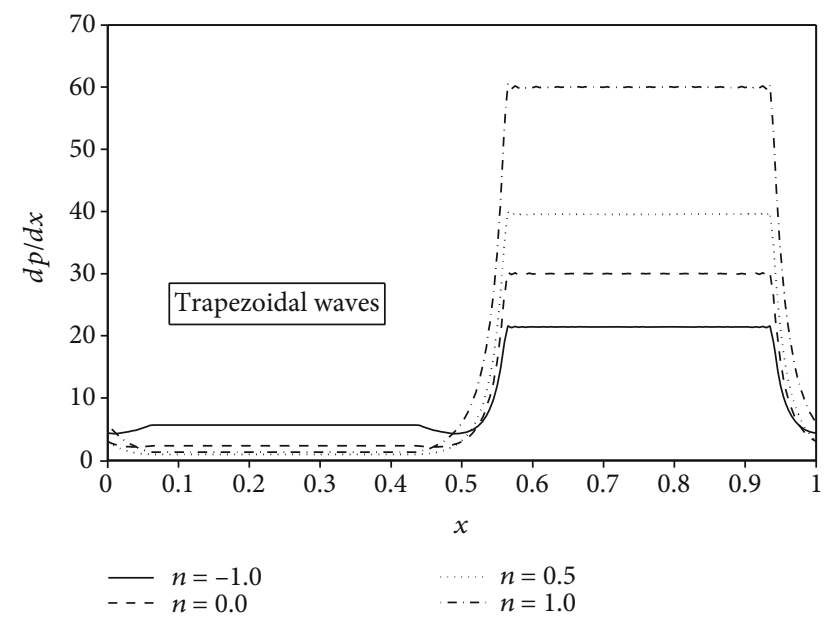

FIGURE 12: Variation of pressure gradient with $x$ for different values of $n$ for fixed values of $\varphi=0.5, \beta=4$, We $=0.6$, and $Q=-2$.

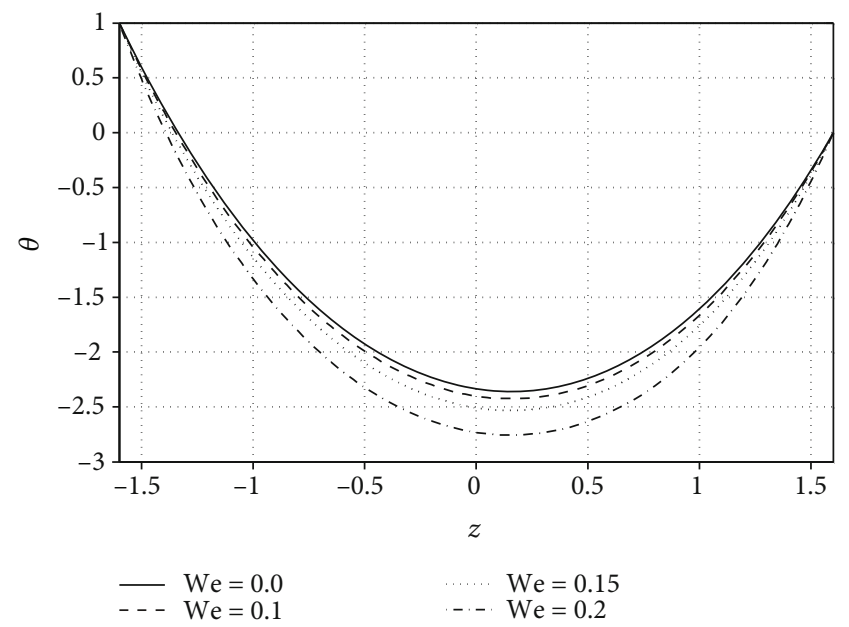

FIgURE 13: Temperature profile for different values of We for fixed values of $\varphi=0.6, x=0, \beta=0.5, n=2, Q=-1, y=1, \operatorname{Pr}=0.5$, and $E c=0.2$.
The resulting series solutions after three iterations are determined using Equation (30) as (when $q \longrightarrow 1$ ) and are evaluated as

$$
\begin{aligned}
& u(x, y, z)=-1+\left(h^{2}-z^{2}\right)+\frac{1-y}{2}-\frac{h-z}{2 h}+\frac{1}{12} \\
& \cdot\left(-12 h^{4}(n-1) \mathrm{We}^{2}+12(n-1) \mathrm{We}^{2} z^{4}\right. \\
& -6 h^{2}\left(2+\frac{d p}{d x}+\frac{1}{4}(n-1) \mathrm{We}^{2} \beta^{2}\right)+6 z^{2} \\
& \left.\cdot\left(2+\frac{d p}{d x}+\frac{1}{4}(n-1) \mathrm{We}^{2} \beta^{2}\right)\right)+\frac{1}{96}(n-1) \mathrm{We}^{2} \\
& \cdot(h-z)(h+z)\left(384(n-1) \mathrm{We}^{2}\left(h^{4}+h^{2} z^{2}+z^{4}\right)\right. \\
& +12(n-1) \beta^{2} \mathrm{We}^{2}\left(h^{2}+z^{2}\right)+6\left(24\left(h^{2}+z^{2}\right)\right. \\
& \left.\left.+\beta^{2}\right)\left(2+\frac{d p}{d x}+\frac{1}{4}(n-1) \mathrm{We}^{2} \beta^{2}\right)\right), \\
& \theta(x, y, z)=-h^{2}+\frac{h-z}{2 h}+z^{2}+\frac{1-y^{2}}{\beta^{2}} \\
& +\frac{1}{60}\left(\operatorname{Pr} E c 16(n-1) \mathrm{We}^{2}\left(h^{6}-z^{6}\right)\right. \\
& +5 \operatorname{Pr} E c\left(h^{4}-z^{4}\right)\left(4+(n-1) \mathrm{We}^{2} \beta^{2}\right) \\
& +\frac{15}{16} \operatorname{Pr} E c(h-z)(h+z) \beta^{2}(8 \\
& \left.\left.+(n-1) \mathrm{We}^{2} \beta^{2}\right)\right)-\frac{1}{1260}(\operatorname{Pr} E c(21(2 \\
& \left.+\frac{d p}{d x}+\frac{1}{4}(n-1) \mathrm{We}^{2} \beta^{2}\right)\left(32 h^{6}(n-1) \mathrm{We}^{2}\right. \\
& +5 h^{4}\left(4+(n-1) \mathrm{We}^{2} \beta^{2}\right) \\
& \left.+z^{4}\left(-20-(n-1) \mathrm{We}^{2}\left(32 z^{2}+5 \beta^{2}\right)\right)\right) \\
& +24(n-1) \mathrm{We}^{2}\left(60 h^{8}(n-1) \mathrm{We}^{2}\right. \\
& +7 h^{6}\left(4+(n-1) \mathrm{We}^{2} \beta^{2}\right) \\
& \left.\left.\left.+z^{6}\left(-28-(n-1) \mathrm{We}^{2}\left(60 z^{2}+7 \beta^{2}\right)\right)\right)\right)\right) \text {. }
\end{aligned}
$$

The volumetric flow rate is given by

$$
\begin{aligned}
q= & \begin{array}{l}
h(x) \\
0
\end{array}{ }_{0}^{1} u(x, y, z) d y d z=-h(x)+\frac{8}{5} h^{5}(n-1) \mathrm{We}^{2} \\
& +\frac{24}{7} h^{7}(n-1)^{2} \mathrm{We}^{4}+\frac{2}{5} h^{5}(n-1)^{2} \beta^{2} \mathrm{We}^{4} \\
& +\frac{1}{96} h^{3}(n-1)^{2} \mathrm{We}^{4} \beta^{4}+\frac{d p}{d x}\left(\frac{6}{5} h^{5}(n-1) \mathrm{We}^{2}\right. \\
& \left.+\frac{1}{24} h^{3}\left(-8+(n-1) \mathrm{We}^{2} \beta^{2}\right)\right) .
\end{aligned}
$$




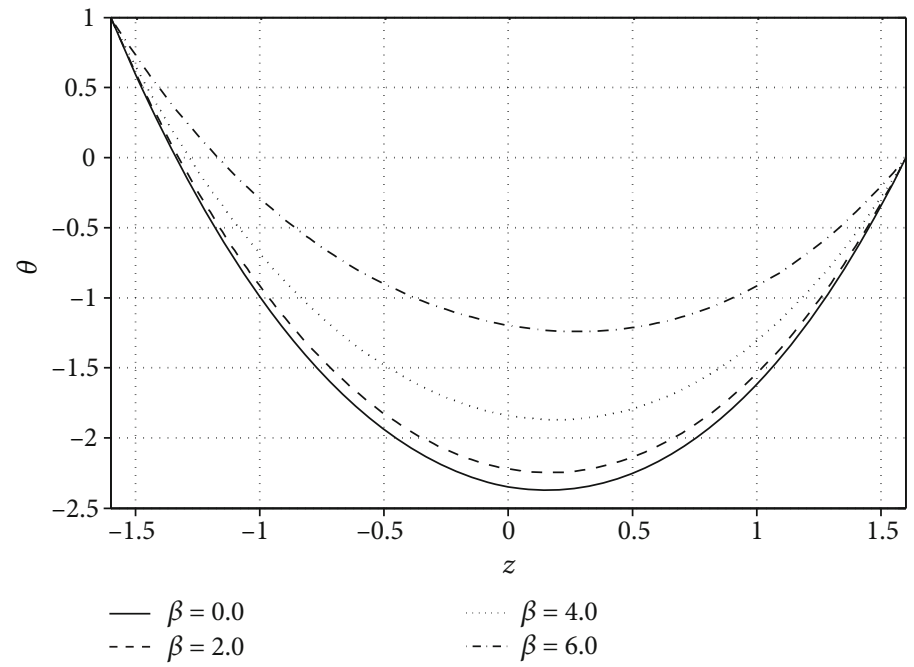

FIGURE 14: Temperature profile for different values of $\beta$ for fixed values of $\varphi=0.6, x=0 \mathrm{We}=0.03, n=2, Q=-1, y=1, \operatorname{Pr}=0.5$, and $E c=0.2$.

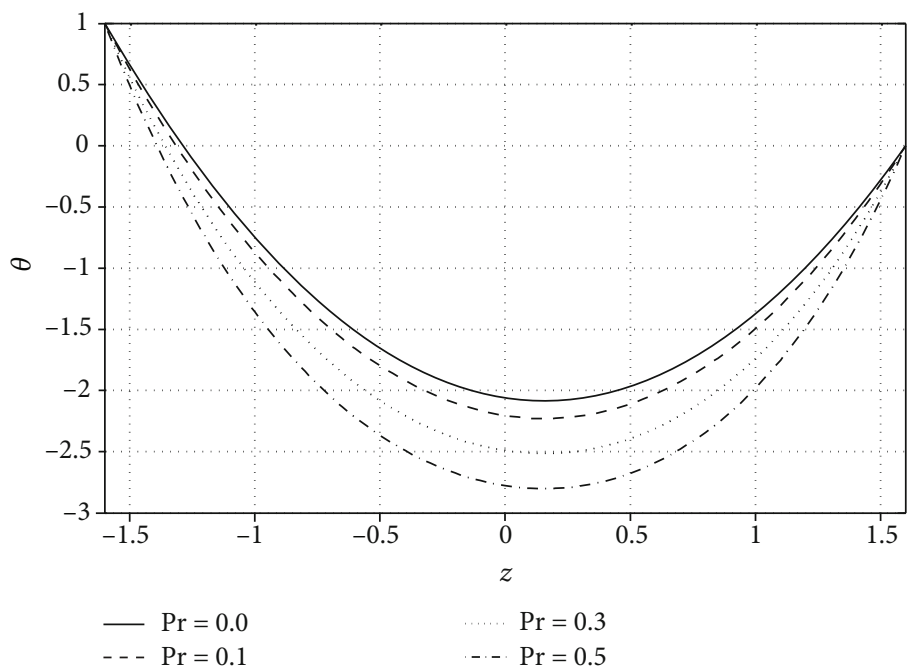

FIGURE 15: Temperature profile for different values of $\operatorname{Pr}$ for fixed values of $\varphi=0.6, x=0$, We $=0.03, n=2, Q=-1, y=1, \beta=0.2$, and $E c=0.5$.

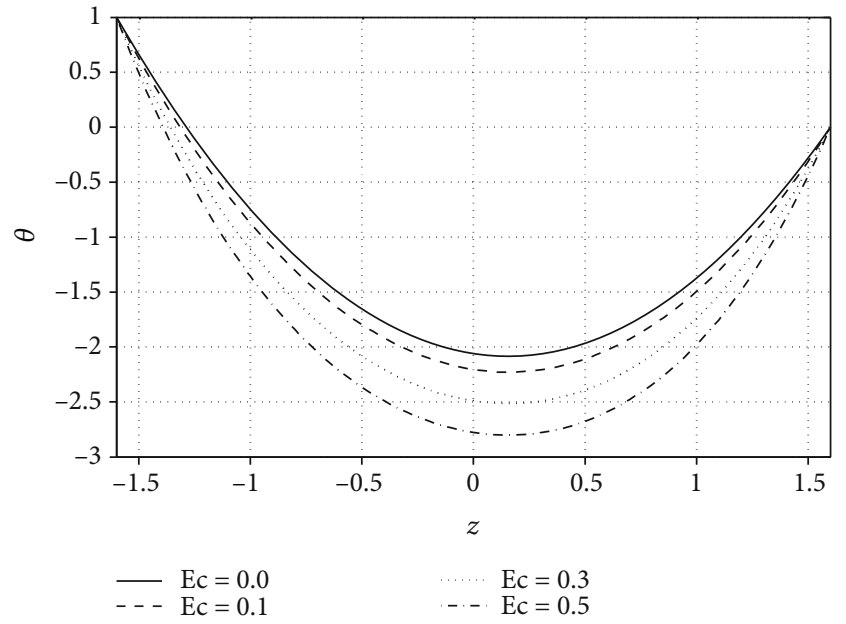

Figure 16: Temperature profile for different values of $E c$ for fixed values of $\varphi=0.6, x=0, \mathrm{We}=0.03, n=2, Q=-1, y=1, \beta=0.2$, and $\operatorname{Pr}=0.5$.

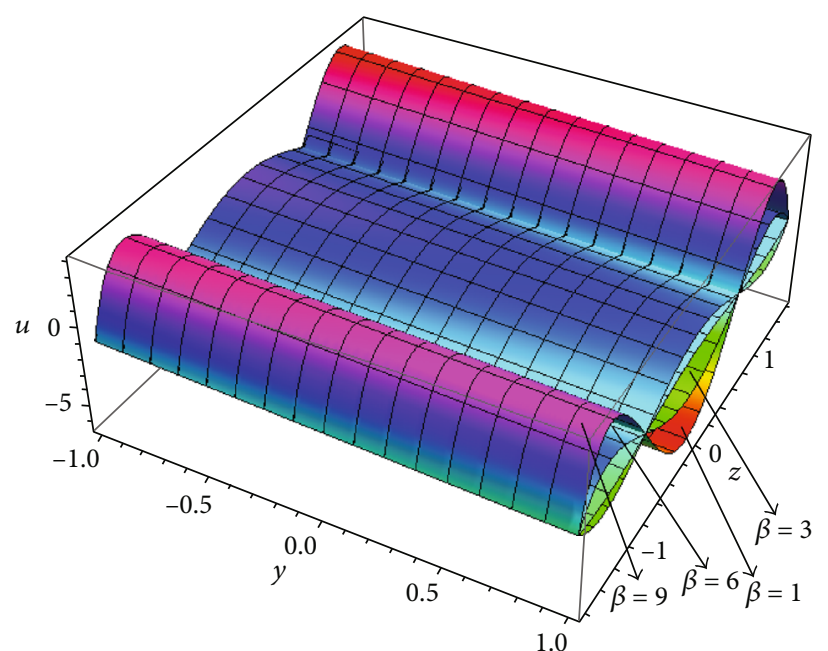

Figure 17: Velocity profile for different values of $\beta$. The other parameters are $\varphi=0.6, x=0, Q=-0.5$, $\mathrm{We}=0.3$, and $n=2$. 


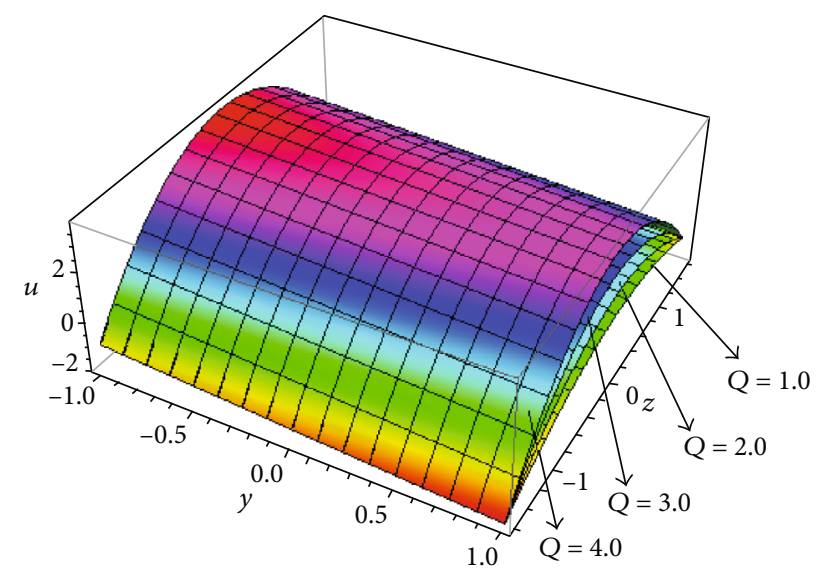

Figure 18: Velocity profile for different values of $Q$. The other parameters are $\varphi=0.6, x=0, \beta=0.5, \mathrm{We}=0.03$, and $n=1$.

The instantaneous flux is defined as

$$
\bar{Q}={ }_{0}^{h(x)}{ }_{0}^{1}(u+1) d y d z=q+h(x) .
$$

The average volume flow rate over one period $(T=\lambda / c)$ of the peristaltic wave is defined as

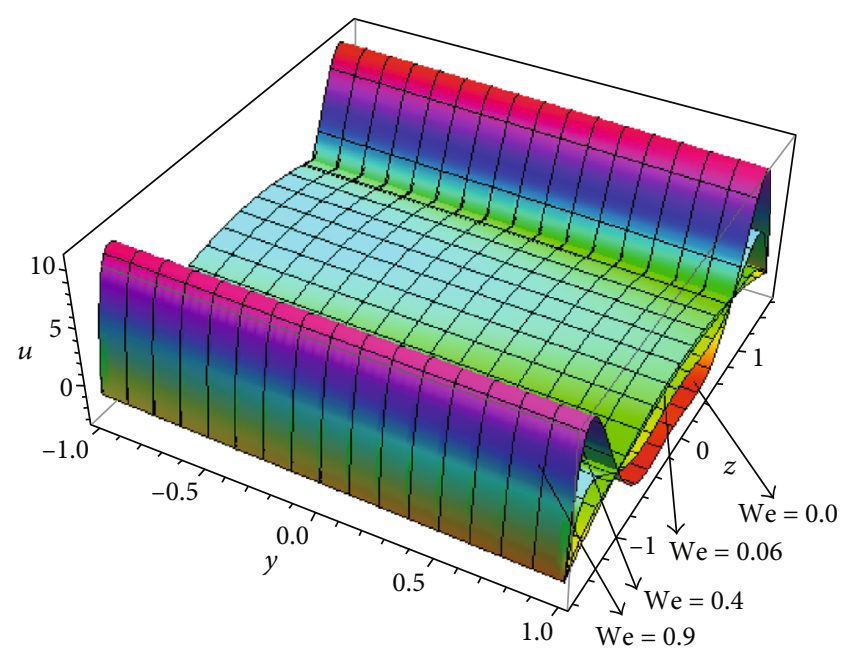

FIgURE 19: Velocity profile for different values of We. The other parameters are $\varphi=0.6, x=0, \beta=0.9, Q=4$, and $n=2$.

$$
Q=\frac{1}{T}_{0}^{T} \bar{Q} d t=q+1
$$

The pressure gradient is obtained from Equations (35) and (37) as

$$
\frac{d p}{d x}=\frac{Q-1+h(x)-(8 / 5) h^{5}(n-1) \mathrm{We}^{2}-(24 / 7) h^{7}(n-1)^{2} \mathrm{We}^{4}-(2 / 5) h^{5}(n-1)^{2} \beta^{2} \mathrm{We}^{4}-(1 / 96) h^{3}(n-1)^{2} \beta^{4} \mathrm{We}}{\left((6 / 5) h^{5}(n-1) \mathrm{We}^{2}+(1 / 24) h^{3}\left(-8+(n-1) \mathrm{We}^{2} \beta^{2}\right)\right)},
$$

Integration of Equation (38) over one wavelength yields

$$
\Delta p={ }_{0}^{1} \frac{d p}{d x} d x
$$

It is noticed here that the limit $\beta \longrightarrow 0$ (keeping it fixed and $d \longrightarrow \infty$ ), the rectangular duct reduces to a twodimensional channel. It is also noticed that when $\beta=1$ the rectangular duct becomes a square duct.

\section{Expressions for Different Wave Shapes}

The nondimensional expressions for five considered wave forms are given by [11]. The expression for the triangular, square, and trapezoidal waves are derived from the Fourier series.

(1) Sinusoidal wave

$$
h(x)= \pm 1 \pm \phi \sin 2 \pi x
$$

(2) Multisinusoidal wave

$$
h(x)= \pm 1 \pm \phi \sin 2 m \pi x
$$

(3) Triangular wave

$$
h(x)= \pm 1 \pm \phi\left[\frac{8}{\pi^{3}} \sum_{m=1}^{\infty} \frac{(-1)^{m+1}}{(2 m-1)^{2}} \sin (2 \pi(2 m-1) x)\right]
$$

(4) Trapezoidal wave

$$
h(x)= \pm 1 \pm \varphi\left[\frac{32}{\pi^{2}} \sum_{m=1}^{\infty} \frac{\sin (\pi / 8)(2 m-1)}{(2 m-1)^{2}} \sin (2 \pi(2 m-1) x)\right]
$$

(5) Square wave

$$
h(x)= \pm 1 \pm \varphi\left[\frac{4}{\pi} \sum_{m=1}^{\infty} \frac{(-1)^{m+1}}{(2 m-1)} \cos (2(2 m-1) \pi x)\right]
$$

\section{Numerical Results and Discussion}

In this segment, the graphical representation of the proposed problem is discussed. With the help of mathematics software 


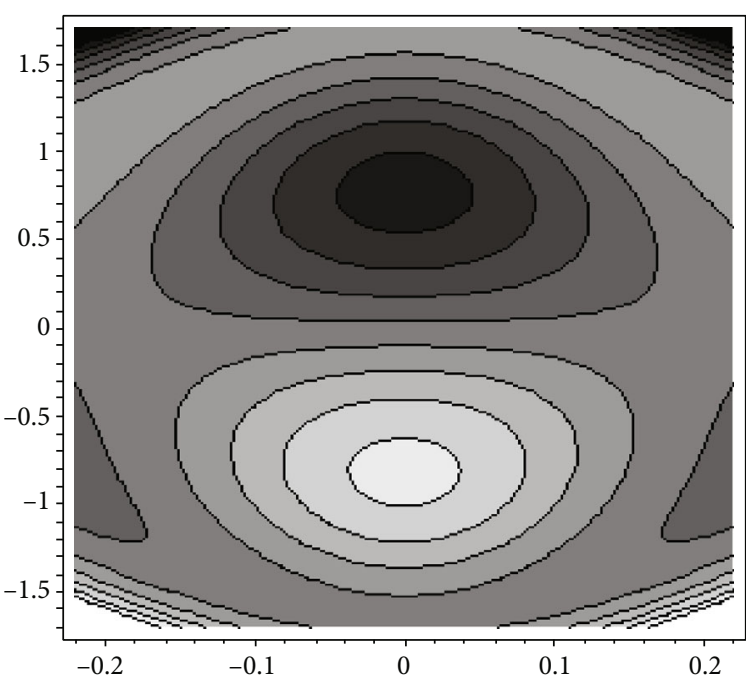

(a)

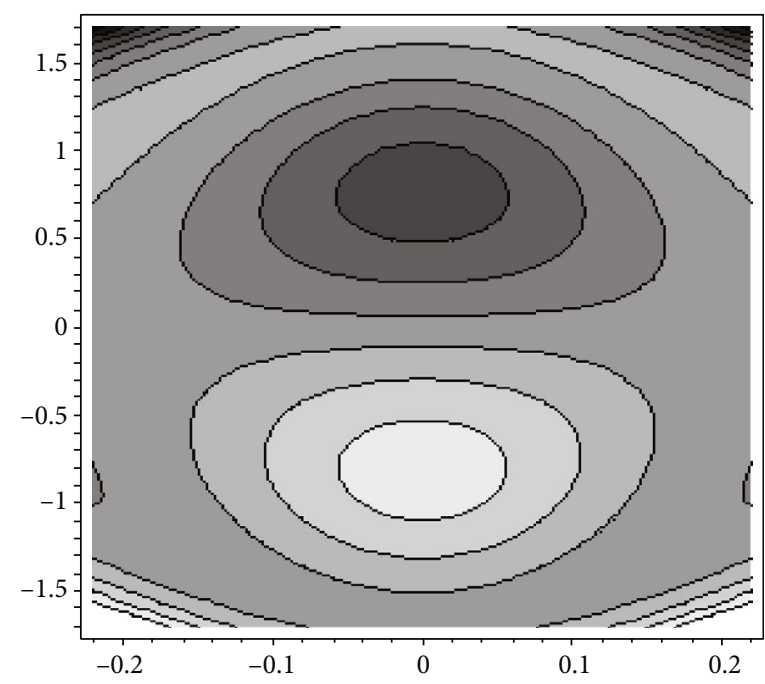

(b)

Figure 20: Stream lines for different values of $\beta$. The other parameters are $\varphi=0.7$, We $=0.51, y=1, n=-0.8$, and $Q=2.0$.

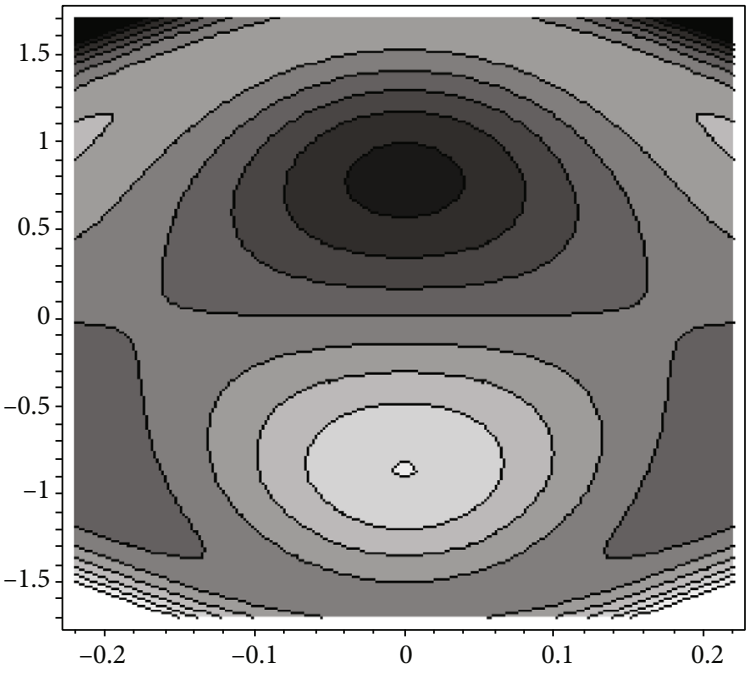

(a)

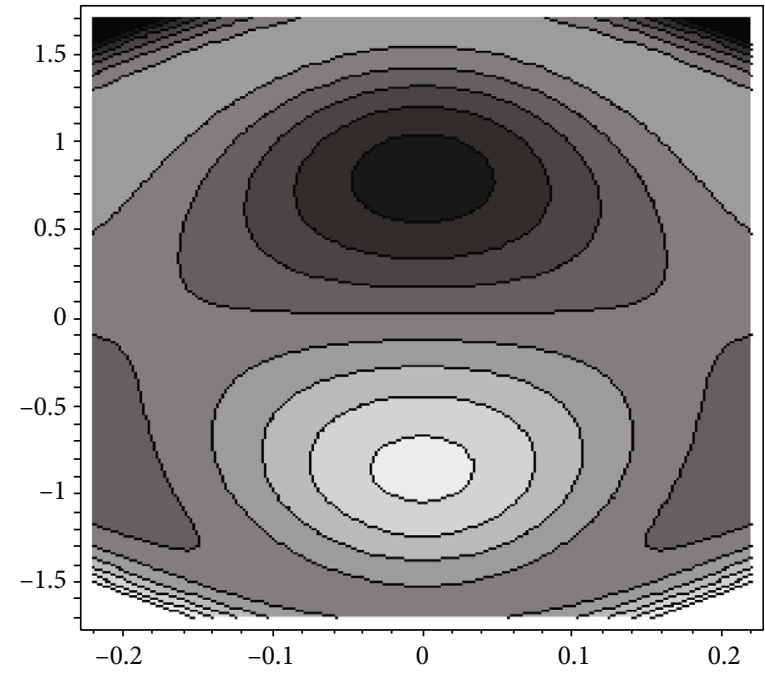

(b)

FIgURE 21: Stream lines for different values of $n$. The other parameters are $\varphi=0.7, \beta=4, \mathrm{We}=0.51, y=1$, and $Q=2.0$.

Mathematica, the expression for pressure rise and pressure gradient is calculated. In order to see the behavior of pressure rise with volume flow rate $Q$ for different values of aspect ratio $\beta$, power law index $n$, amplitude ratio $\phi$, and Weissenberg number We. It is observed from Figure 1 that in the peristaltic pumping $(\Delta p>0, Q>0)$ and retrograde pumping $(\Delta p>0, Q<0)$ regions, the pumping rate decreases with an increase in aspect ratio $\beta$, while in the copumping region $\left(\Delta_{p}<0, Q>0\right)$, the behavior is quite opposite; here, the pumping rate increases with an increase in aspect ratio $\beta$. Figures 2 and 3 show the variation of pressure rise with volume flow rate $Q$ for different values of $n$ and $\phi$. It is observed from Figure 2 that in the retrograde pumping $(\Delta p>0, Q>0)$ and peristaltic pumping $(\Delta p>0, Q<0)$ regions, the pumping rate increases with an increase in $n$ and $\phi$, while in the copumping region $\left(\Delta_{p}<0, Q>0\right)$, the pressure rise decreases with an increase in values of $n$ and $\varphi$. In order to see the behavior of pressure rise with volume flow rate $Q$ for different values of We, Figure 4 is plotted. It is depicted from Figure 4 that in the peristaltic pumping $(\Delta p>0, Q>0)$ and retrograde pumping $(\Delta p>0, Q<0)$ regions, the pumping rate decreases with an increase in We, while in the copumping region $\left(\Delta_{p}<0, Q>0\right)$, the behavior is quite opposite; here, the pumping rate increases with an increase in We. The pressure gradient for different values of aspect ratio $\beta$, $n$, amplitude ratio $\phi$, and We against space variable $x$ is plotted in Figures 5-8. It is depicted from Figures 5-8 that for $x \in[0,0.2]$ and $x \in[0.8,1]$, the pressure gradient is small, i.e., the flow can easily pass without the imposition of a large pressure gradient, while in the region $x \in[0.2,0.8]$ that the pressure gradient decreases with an increase in aspect ratio $\beta$ and We and increases with an increase in amplitude ratio $\phi$ 


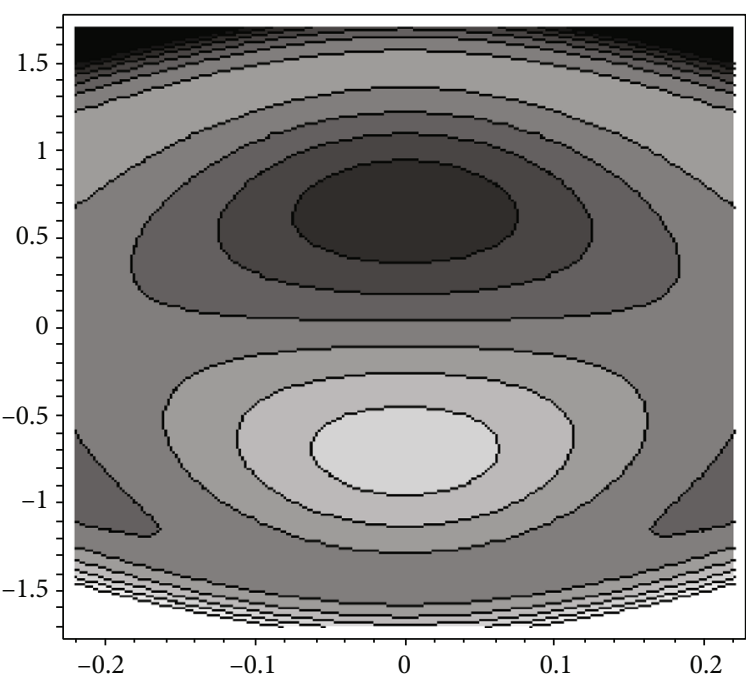

(a)

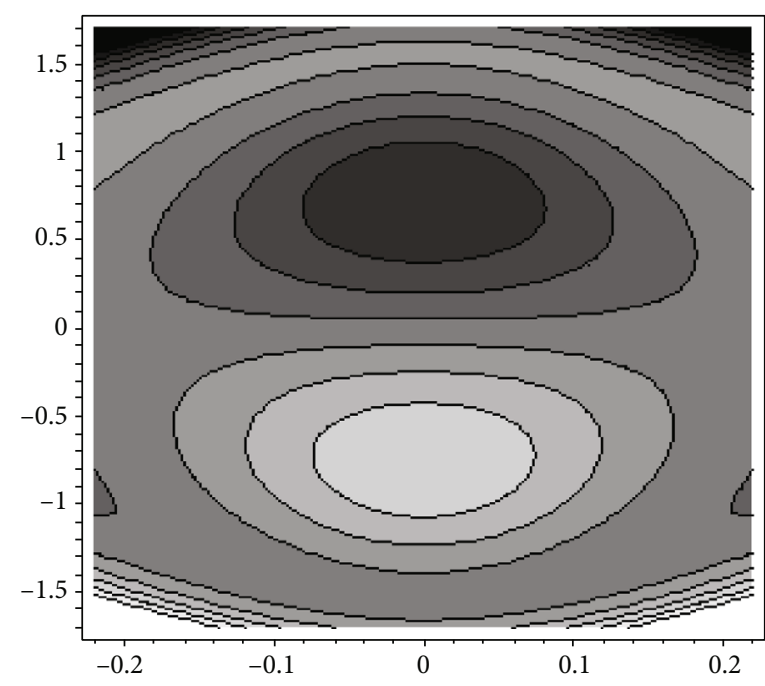

(b)

FIgURE 22: Stream lines for different values of $\varphi$. The other parameters are $n=-0.8, \beta=7, \mathrm{We}=0.6, y=1$, and $Q=2.0$.

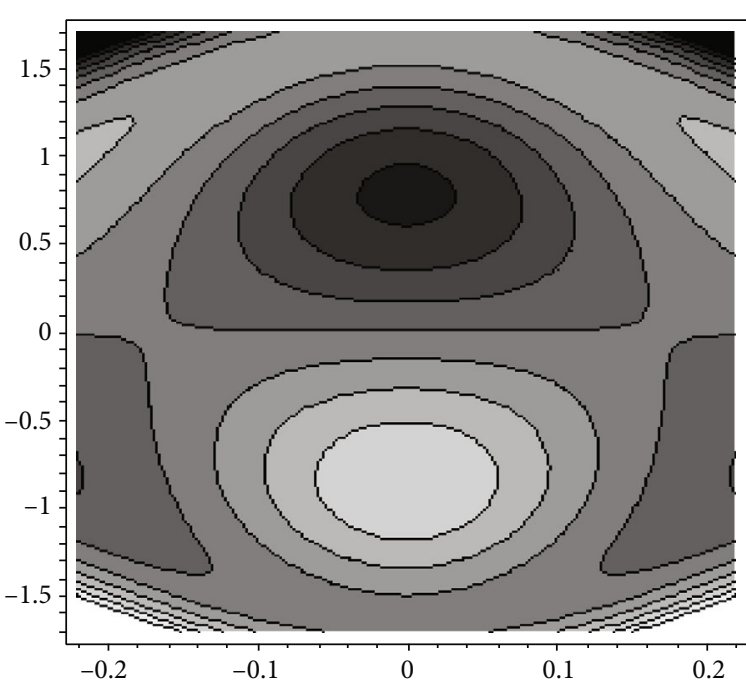

(a)

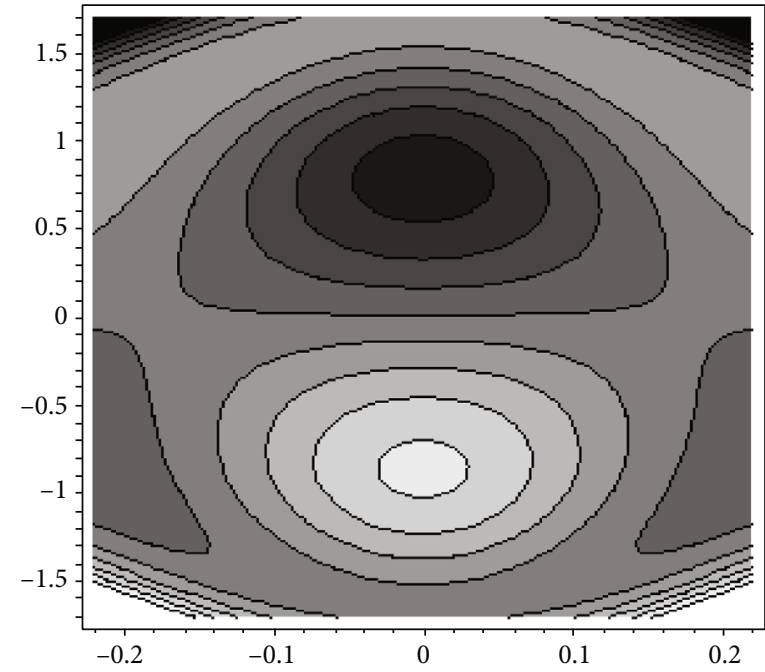

(b)

FIgURE 23: Stream lines for different values of We. The other parameters are $n=-0.8, \beta=4, \varphi=0.7, y=1$, and $Q=2.0$.

and $n$, so much pressure gradient is required to maintain the flux to pass. Figures $9-12$ show the stream lines for different wave forms. It is observed from Figures 9-12 that the pressure gradient retains the same shape of the waves as we consider.

Figures 13-16 show the temperature profile for different values of $\mathrm{We}, \beta, \mathrm{Pr}$, and $E c$. It is depicted from Figure 13 that the magnitude value of the temperature profile increases with an increase in We. It is observed from Figure 14 that the magnitude value of the temperature profile decreases with an increase in $\beta$ Figures 15 and 16 show the temperature profile for different values of $\operatorname{Pr}$ and $E c$. It is observed from Figures 15 and 16 that temperature profile increases with an increase in $\operatorname{Pr}$ and $E c$. Velocity profile for different values of $\beta, Q$, and We is plotted in Figures 17-19. It is observed from Figures 17 and 18 that the magnitude value of the velocity profile decreases with an increase in $\beta$ volume flow rate $Q$. It is depicted from Figure 19 that the magnitude value of the velocity profile increases with an increase in We. Stream lines for different values of aspect ratio $\beta$ amplitude ratio $\phi$, power law index $n$, and Weissenberg number We is plotted in Figures 20-23. It is observed from Figure 20 that the number of the trapping bolus decreases with an increase in aspect ratio $\beta$. It is observed from Figures 21 and 22 that the size of the trapping bolus increases with an increase in power law index $n$ and amplitude ratio $\phi$ Figure 23 shows the stream lines for different values of the Weissenberg number We. It is depicted from Figure 23 that the number of the trapping bolus increases in the lower half of the channel with an increase in We, while in the upper half of the channel the size 


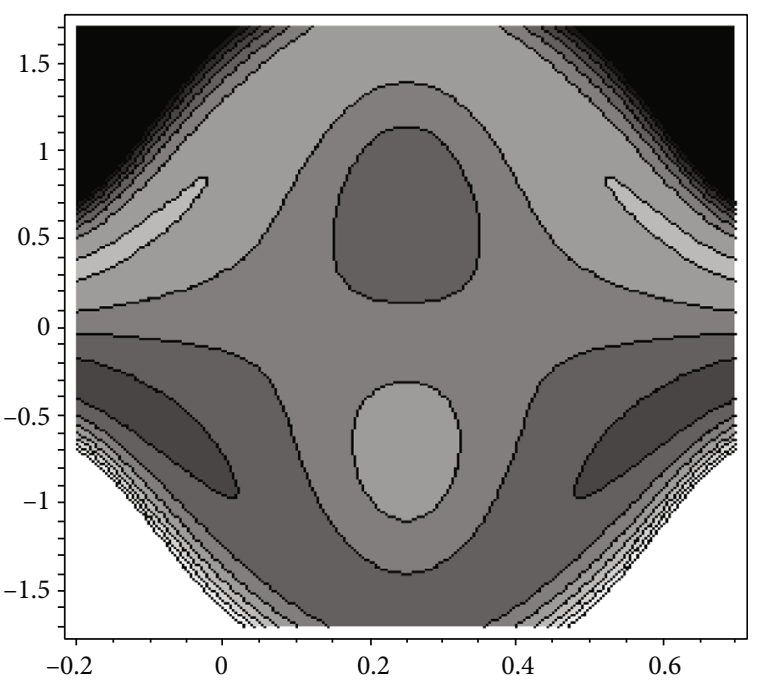

(a)

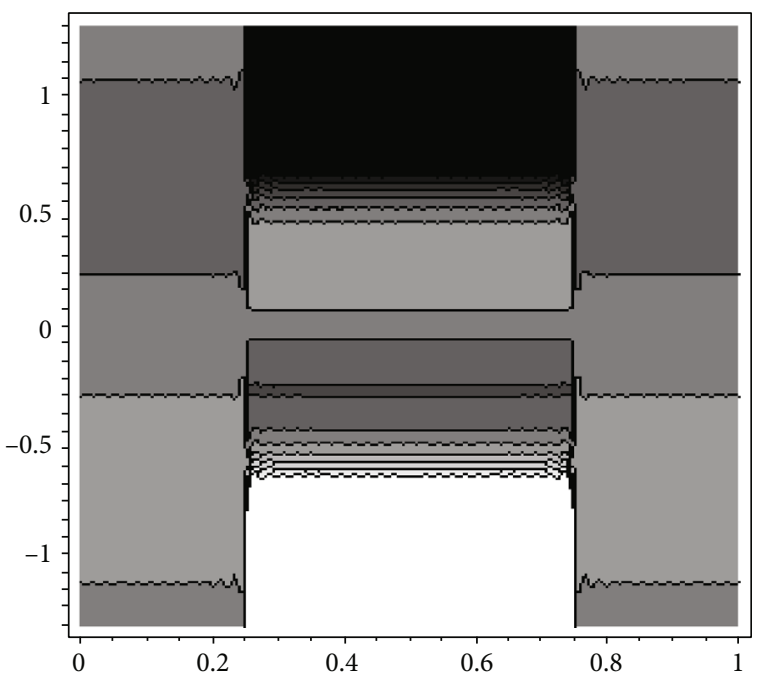

(c)

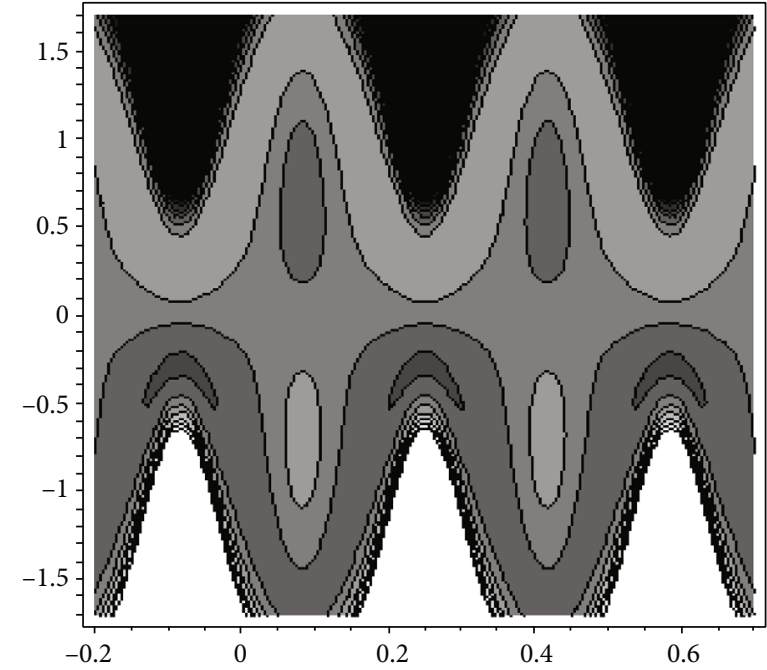

(b)

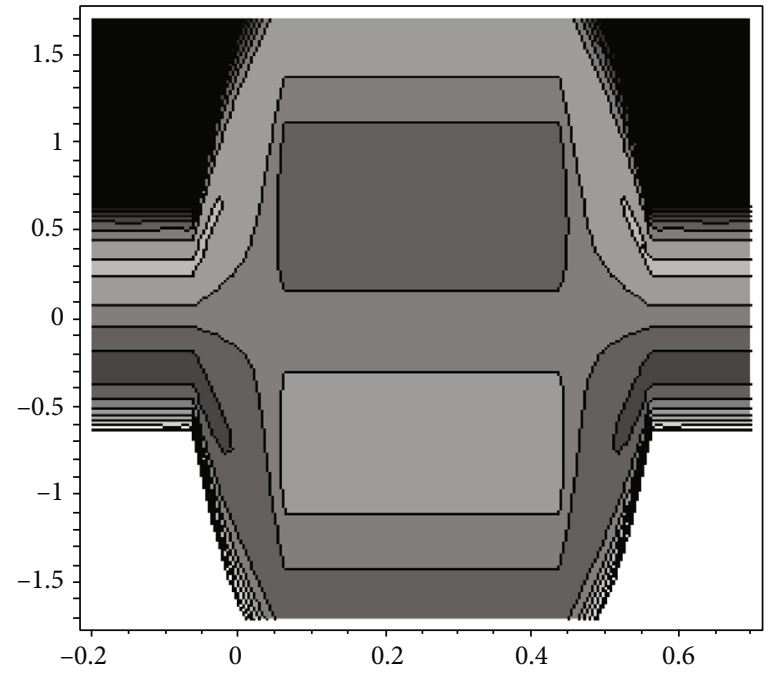

(d)

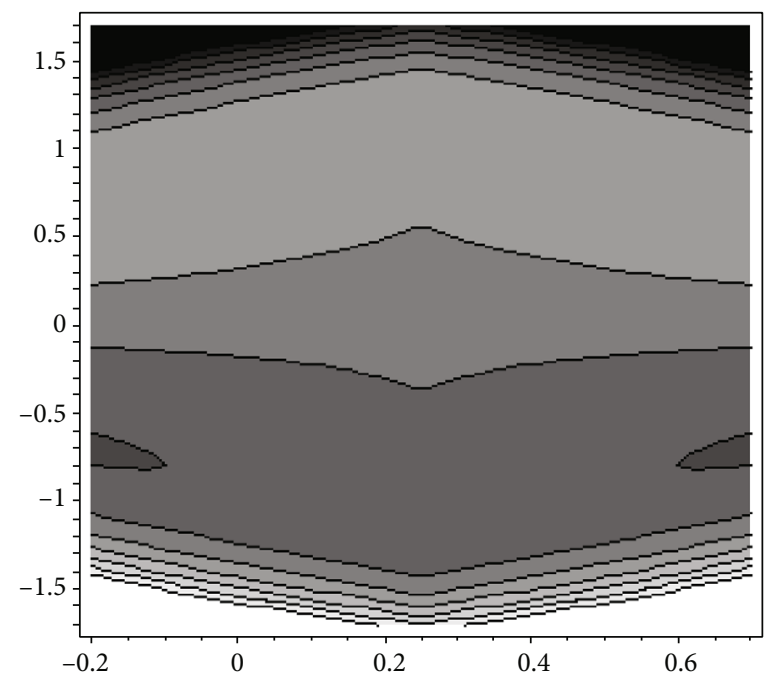

(e)

Figure 24: Stream lines for different wave forms (a) for the sinusoidal wave, (b) for the multisinsoidal wave, (c) for the square wave, (d) for the trapezoidal wave, and (e) for the triangular wave. The other parameters are $n=-0.8, \beta=6, \varphi=0.7, y=1, \mathrm{We}=0.53$, and $Q=3.3$. 
of the trapping bolus increases. Figure 24 shows the stream lines for different wave forms.

\section{Conclusion}

We have discussed the impact of heat transfer on peristaltic flow of a Carreau fluid in a rectangular duct with different wave forms. The governing equations for a threedimensional rectangular channel are the first model for Carreau fluid and then simplified under long wave length and low Reynolds number approximation. The analytical solutions of highly nonlinear equations are calculated. The results are discussed through graphs. It is observed that pumping rate decreases with an increase in aspect ratio $\beta$ and We in the peristaltic $(\Delta p>0, Q>0)$ and retrograde pumping $(\Delta p>0, Q<0)$ regions, while in the copumping region $(\Delta p<0, Q>0)$, the behavior is quite opposite. It is also noted that in the retrograde pumping $(\Delta p>0, Q<0)$ and peristaltic pumping $(\Delta p>0, Q$ $>0)$ regions, the pumping rate increases with an increase in $n$ and $\varphi$. It is observed that the profile of temperature increases with an increase in $\mathrm{We}, \mathrm{Pr}$, and $E c$, while temperature profile shows the opposite behavior in the case of $\beta$. It is also observed that number of trapping bolus increases in the lower half of the channel with an increase in We, while in the upper half of the channel, size of the trapping bolus increases.

\section{Nomenclature}

$a, b:$ Amplitudes of waves

$c$ : Velocity of propagation

$X: \quad$ Direction of wave propagation

$C^{\prime}$ : Specific heat

T: Temperature

$\delta$ : $\quad$ Dimensionless wave number

$\beta$ : Aspect ratio

We: Weissenberg number

$n$ : $\quad$ Power law index

$\theta: \quad$ Temperature of fluid in dimensionless form

$\lambda$ : Wavelength

$t$ : Time

$\rho:$ Density

$P$ : $\quad$ Pressure

Re: Reynolds number

Q: Volume flow rate

Pr: Prandtl number

$\varphi$ : Amplitude ratio

Ec: Eckert number

S: $\quad$ Stress tensor for Carreau fluid.

\section{Data Availability}

Data used to support the findings of this work are included in the article.

\section{Conflicts of Interest}

The authors declare no conflict of interest.

\section{Acknowledgments}

The authors would like to acknowledge the support provided by the Deanship of Scientific Research (DSR) at the Prince Mohammad Bin Fahd University (PMU) for funding this work.

\section{References}

[1] T. W. Latham, Fluid motion in a peristaltic pump, [M.S. thesis], MIT, Cambridge, 1996.

[2] Y. Abd elmaboud, S. I. Abdelsalam, and K. S. Mekheimer, "Couple stress fluid flow in a rotating channel with peristalsis," Journal of Hydrodynamics, vol. 30, no. 2, pp. 307-316, 2018.

[3] A. Riaz, A. Zeeshan, M. M. Bhatti, and R. Ellahi, "Peristaltic propulsion of Jeffrey nano-liquid and heat transfer through a symmetrical duct with moving walls in a porous medium," Physica A: Statistical Mechanics and its Applications, vol. 545, 2020.

[4] S. Akram, S. Nadeem, and M. Hanif, "Numerical and analytical treatment on peristaltic flow of Williamson fluid in the occurrence of induced magnetic field," Journal of Magnetism and Magnetic Materials, vol. 346, pp. 142-151, 2013.

[5] S. Nadeem and S. Akram, "Magnetohydrodynamic peristaltic flow of a hyperbolic tangent fluid in a vertical asymmetric channel with heat transfer," Acta Mechanica Sinica, vol. 27, no. 2, pp. 237-250, 2011.

[6] A. Riaz, A. Zeeshan, S. Ahmad, A. Razaq, and M. Zubair, "Effects of external magnetic field on non-newtonian two phase fluid in an annulus with peristaltic pumping," Journal of Magnetics, vol. 24, no. 1, pp. 62-69, 2019.

[7] S. Nadeem and S. Akram, "Peristaltic flow of a Maxwell model through porous boundaries in a porous medium," Transport in Porous Media, vol. 86, no. 3, pp. 895-909, 2011.

[8] R. Ellahi, A. Riaz, S. Nadeem, and M. Mushtaq, "Series solutions of magnetohydrodynamic peristaltic flow of a Jeffrey fluid in eccentric cylinders," Applied Mathematics \& Information Sciences, vol. 7, no. 4, pp. 1441-1449, 2013.

[9] S. Nadeem and S. Akram, "Peristaltic flow of a couple stress fluid under the effect of induced magnetic field in an asymmetric channel," Archive of Applied Mechanics, vol. 81, no. 1, pp. 97-109, 2011.

[10] A. H. Shapiro, M. Y. Jaffrin, and S. L. Weinberg, "Peristaltic pumping with long wavelengths at low Reynolds number," Journal of Fluid Mechanics, vol. 37, no. 4, pp. 799-825, 1969.

[11] A. Riaz, H. Alolaiyan, and A. Razaq, "Convective heat transfer and magnetohydrodynamics across a peristaltic channel coated with nonlinear nanofluid," Coatings, vol. 9, no. 12, p. 816, 2019.

[12] A. Riaz, S. U. D. Khan, A. Zeeshan, S. U. Khan, M. Hassan, and T. Muhammad, "Thermal analysis of peristaltic flow of nanosized particles within a curved channel with second-order partial slip and porous medium," Journal of Thermal Analysis and Calorimetry.

[13] H. Alolaiyan, A. Riaz, A. Razaq, N. Saleem, A. Zeeshan, and M. M. Bhatti, "Effects of double diffusion convection on third grade nanofluid through a curved compliant peristaltic channel," Coatings, vol. 10, no. 2, p. 154, 2020.

[14] Y. A. S. El-Masry, Y. Abd Elmaboud, and M. A. Abdel-Sattar, "The impacts of varying magnetic field and free convection 
heat transfer on an Eyring-Powell fluid flow with peristalsis: VIM solution," Journal of Taibah University for Science, vol. 14, no. 1, pp. 19-30, 2020.

[15] Y. A. S. El-Masry, Y. Abd Elmaboud, and M. A. Abdel-Sattar, "Direct current/alternating current magnetohydrodynamic micropump of a hybrid nanofluid through a vertical annulus with heat transfer," Journal of Thermal Science and Engineering Applications, vol. 12, no. 4, 2020.

[16] M. V. Subba Reddy, M. Mishra, S. Sreenadh, and A. Ramachandra Rao, "Influence of lateral walls on peristaltic flow in a rectangular duct," Journal of Fluids Engineering, vol. 127, no. 4, pp. 824-827, 2005.

[17] S. Nadeem, S. Akram, T. Hayat, and A. A. Hendi, "Peristaltic flow of a Carreau fluid in a rectangular duct," Journal of Fluids Engineering, vol. 134, no. 4, 2012.

[18] R. Ellahi, F. Hussain, F. Ishtiaq, and A. Hussain, "Peristaltic transport of Jeffrey fluid in a rectangular duct through a porous medium under the effect of partial slip: an application to upgrade industrial sieves/filters," Pramana, vol. 93, no. 3, 2019.

[19] S. Nadeem and S. Akram, "Peristaltic flow of a Jeffrey fluid in a rectangular duct," Nonlinear Analysis: Real World Applications, vol. 11, no. 5, pp. 4238-4247, 2010.

[20] S. Akram, K. S. Mekheimer, and Y. A. Elmaboud, "Particulate suspension slip flow induced by peristaltic waves in a rectangular duct: effect of lateral walls," Alexandria Engineering Journal, vol. 57, no. 1, pp. 407-414, 2018.

[21] A. Riaz, R. Ellahi, M. M. Bhatti, and M. Marin, "Study of heat and mass transfer in the Eyring-Powell model of fluid propagating peristaltically through a rectangular compliant channel," Heat Transfer Research, vol. 50, no. 16, pp. 1539-1560, 2019.

[22] R. Ellahi, A. Riaz, and S. Nadeem, "A theoretical study of Prandtl nanofluid in a rectangular duct through peristaltic transport," Applied Nanoscience, vol. 4, no. 6, pp. 753-760, 2014.

[23] S. Akram, K. S. Mekheimer, and S. Nadeem, "Influence of lateral walls on peristaltic flow of a couple stress fluid in a non-uniform rectangular duct," Applied Mathematics \& Information Sciences, vol. 8, no. 3, pp. 1127-1133, 2014.

[24] D. D. Ganji and A. Rajabi, "Assessment of homotopy-perturbation and perturbation methods in heat radiation equations," International Communications in Heat and Mass Transfer, vol. 33, no. 3, pp. 391-400, 2006.

[25] D. D. Ganji and A. Sadighi, "Application of He's Homotopyperturbation method to nonlinear coupled Systems of reaction-diffusion equations," International Journal of Nonlinear Sciences and Numerical Simulation, vol. 7, no. 4, pp. 411418, 2006.

[26] D. Lu, A. Seadawy, and M. Arshad, "Applications of extended simple equation method on unstable nonlinear Schrödinger equations," Optik, vol. 140, pp. 136-144, 2017.

[27] M. Arshad, D. Lu, M.-U. Rehman, I. Ahmed, and A. M. Sultan, "Optical solitary wave and elliptic function solutions of the Fokas-Lenells equation in the presence of perturbation terms and its modulation instability," Physica Scripta, vol. 94, no. 10, 2019.

[28] A. Saadatmandi, M. Dehghan, and A. Eftekhari, "Application of He's homotopy perturbation method for non-linear system of second-order boundary value problems," Nonlinear Analy- sis: Real World Applications, vol. 10, no. 3, pp. 1912-1922, 2009.

[29] J. H. He, "Homotopy perturbation method for solving boundary value problems," Physics Letters A, vol. 350, no. 1-2, pp. 8788, 2006.

[30] J. H. He, "A note on the homotopy perturbation method," Thermal Science, vol. 14, pp. 565-568, 2010. 